\title{
ANALYSIS OF PHOTOLUMINISCENCE IN THE nCSi-DMA SYSTEM
}

Silicon nanocrystalline particles (ncSi) were fabricated from the Si swarf using the beads milling method. Observed photoluminiscence spectra (PL) of the ncSi in hexane with the dimethylanthracene molecules (DMA) show photoluminescence peaks at energies of 2.55, 2.75, 2.92, and $3.09 \mathrm{eV}$. The shape of PL spectra corresponds to the vibronic structure of adsorbed DMA molecules. The PL intensity of the ncSi-DMA system increases by 3000 times by adsorption of DMA on Si nanoparticles. The PL enhancement results from an increase in absorption probability of incident light by DMA caused by adsorption on the surface of $n c S i$. Theoretical model of the PL experiment was constructed and resulting model parameters were used in analysis of possible PL transitions and charge transfer processes.

Keywords: Semiconductor, silicon nanocrystal, DMA, photoluminiscence.

\section{Introduction}

The properties of many materials change when formed from nanoparticles. Nanoparticles have one dimension reaching 100 nanometers or less. Nanoparticles may exhibit size-related properties that differ significantly from those observed in fine particles or bulk materials. Greater surface area of nanoparticle causes higher reactivity to some other molecules as well as increased ability to adsorb and carry other compounds. The reactivity of the surface originates from quantum phenomena. Immediately after the creation, nanoparticles may have their surface modified due to the presence of reactants and adsorbing compounds. Due to the unique properties nanocrystals and other nanoparticles (quantum dots, nanobars, dendrimers, nanoshells and similar) have been receiving important attention for potential applications in various areas - from optical and electronic to biology and medicine [1 - 4].

In the present study, silicon nanoparticles have been fabricated from swarf (industrial waste produced during slicing $\mathrm{Si}$ ingots) by the use of the beads milling method [5, 6]. Si nanoparticles can be also formed by various different methods - laser ablation [7, 8], plasma-enhanced chemical vapor deposition (CVD) [9], hot wire CVD [10], sputtering [11], Si implantation [12], intense pulsed ion beam evaporation [13], and other methods. These methods require vacuum conditions and are time- and cost-consuming in comparison to the beads milling method. The size of $\mathrm{Si}$ nanoparticles is modified by oxidation and produced nanoparticle system shows increased photoluminiscence. Produced Si nanoparticles show PL spectra with structure which is attributable to the vibronic structure of 9,10-dimethylanthracene molecule, $\mathrm{C} 16 \mathrm{H} 14$, adsorbed on the nanoparticle Si surface from the hexane environment. Due to the increased probability of excitation of adsorbed DMA molecule the PL intensity of formed system is increased by $\sim 3000$ times. We analysed possible excitation processes leading to modification of experimental PL spectra of ncSi-DMA system. We created theoretical PL model based on modelling of proposed photon emission transitions in the ncSi-DMA system. Theoretical model was optimized in comparison to the experimental PL spectra in order to extract reliable information about the optical excitation and charge transport processes in studied ncSi-DMA system.

\section{Experimental}

Si nanoparticles were produced by using the beads milling method. Milling with $0.5-\mathrm{mm}$ zirconia beads followed by 0.3-mm zirconia beads was performed in 2-propanol. Fabricated ncSi particles were filtered by the use of a teflon membrane filter, and immersed in hexane with DMA molecules. The PL spectra measurements were carried out with a JASCO FP-8500 spectrometer after removal of aggregated and precipitated $\mathrm{Si}$ nanoparticles. Several excitation energies were used in the PL experiments.

\footnotetext{
* ${ }^{1}$ Stanislav Jurecka, ${ }^{2}$ Kentaro Imamura, ${ }^{2}$ Taketoshi Matsumoto, ${ }^{2}$ Hikaru Kobayashi

${ }^{1}$ Institute of Aurel Stodola, University of Zilina, Liptovsky Mikulas, Slovakia

${ }^{2}$ Institute of Scientific and Industrial Research, Osaka University and CREST, Japan Science and Technology Agency, Ibaraki, Japan

E-mail: jurecka@lm.uniza.sk
} 


\section{Results and discussion}

Experimental PL spectra of the ncSi-DMA system are shown in Fig. 1. The shape of PL spectra is strongly influenced by the excitation energy. For excitation energies under $4 \mathrm{eV}$ the PL peaks are determined mainly by the the vibronic structure corresponding to transitions between excited states in the DMA molecules with peak positions near 2.55, 2.75, 2.92, and $3.09 \mathrm{eV}$ [14]. Similar PL experiments were also performed by using ncSi in clean 2, 2, 4-trimethyl pentane (without DMA molecules), resulting in PL spectra without the DMA contribution. The vibronic structure arises therefore from the DMA molecules adsorbed on Si nanoparticles, and the PL intensity is enhanced due to the excitation processes in the ncSi-DMA system. For PL excitation energies above $4 \mathrm{eV}$ the shape of PL spectra drastically changes. It is caused by additional photon and charge interactions in studied system. Peak structure corresponding to the DMA excited states is also observed in this case.

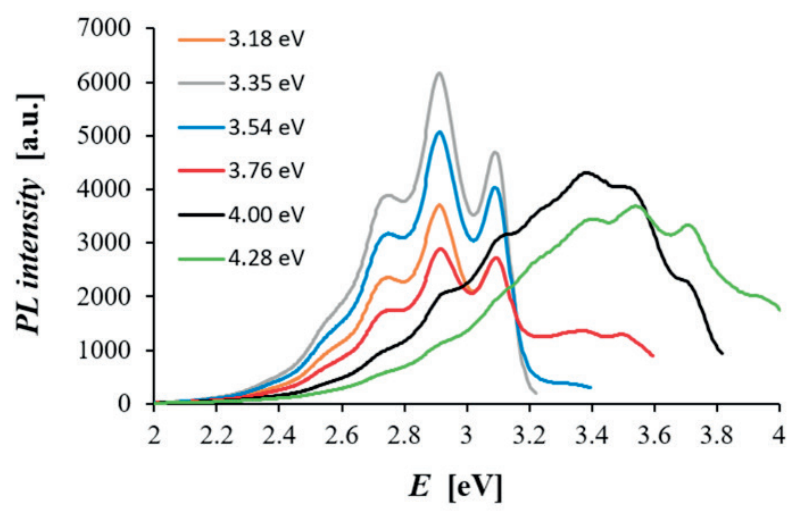

Fig. 1 Experimental PL spectra of the ncSi-DMA system for a set of excitation energies 3.18, 3.35, 3.54, 3.76, 4.00 and $4.28 \mathrm{eV}$

For description of the structure of energy states in the ncSi-DMA system contributing to the PL transitions we expect excitation of following subsystems: a) ncSi excitons, b) DMA optical excitation, c) dipole-dipole optical excitation of DMA molecules from excitons confined in ncSi (energy transfer), d) energy and charge transfer between excitons in ncSi and DMA molecules (diffuse contact of subsystems). DMA is a molecule with a singlet ground state $S_{0}$ and triplet first excited state $T_{1}$ . Dipole interaction based on optically allowed transitions in donor (ncSi) - acceptor (DMA) system is forbidden due to the spin-flip rules for $T_{1}-S_{0}$ transitions. Singlet-triplet splitting energy for the DMA molecule is $1.73 \mathrm{eV}$ and therefore the DMA subsystem cannot be efficiently optically excited by $E_{e x}>1.73$ $\mathrm{eV}$. The direct charge exchange in the ncSi-DMA system is enabled excitation process, triplet exciton annihilation in DMA accompanied by spin-flip is allowed (for $\mathrm{ncSi}$ spin restrictions are lifted). For modelling of the PL spectra we constructed theoretical model considering all the excitations mentioned above. It is based on the superposition of Lorentz functions for description of direct charge exchange processes in donor-acceptor system (from 4 to 8 Lorentz functions). Contribution of confined ncSi excitons is modelled in our approach by using a Gaussian function. We suppose excitation of $\mathrm{ncSi}$ nanocrystals with certain size distribution, determined by the milling procedure. Motivation for using Gaussian model for this PL process is based on superposition of a large number of independent contributions from individually excited Si nanocrystals according to the central limit theorem. PL quenching of optical excitation in donoracceptor system is also supposed. Lorentz function $I_{L}$ is defined by the equation

$I_{L}(x)=\frac{I_{0 L} w^{2}}{\left(x-x_{0}\right)^{2}+w^{2}}$,

where $x_{0}$ is the central position of the peak function, $I_{0 L}$ is the peak intensity, and $w$ is the width parameter. Gauss function is defined by the equation

$I_{G}(x)=\frac{I_{0 G}}{\sqrt{2 \pi} \sigma} \exp \left\{-\left(x-x_{0}\right)^{2} / \sigma^{2}\right\}$,

where $I_{0 G}$ is the peak intensity and $\sigma$ is the width parameter. Experimental PL spectrum is modelled by the theoretical model

$I_{P L}(x)=I_{G}(x)+\sum_{i=1}^{n} I_{L, i}(x)$,

where $x$ is photon energy in $\mathrm{eV}$, and $n=4,8$ for construction of theoretical PL model with 4 or 8 Lorentz functions. The proposed theoretical model is optimized in a comparison with the experimental data by using two step procedure. In the first step, the initial estimation of model parameters is performed by visual modelling, consisting in a modification of model parameters in graphical user interface in order to achieve relatively good agreement between theoretical and experimental PL spectrum. In the next step, this initial estimation is refined by using genetic algorithm combined with Marquardt-Levenberg optimization step $[15,16]$. Coefficient of determination $r^{2}$ [17], describing the quality of optimized theoretical model, typically reaches values at 0.9999 level. Finally, optimal values of model parameters describing positions and shape characteristics for used peak functions are extracted and used for an interpretation of PL excitation processes.

In Fig. 2 optimized theoretical models based on one Gaussian function and four Lorentz functions for excitation energies a) $E_{e x}=3.54 \mathrm{eV}$ and b) $E_{e x}=3.76 \mathrm{eV}$ are shown.

Modelling of PL transmission processes based on selected excitation mechanisms provides theoretical model of the PL experiments in good agreement with experimental data. Without accounting for the contribution of the ncSi distribution to the PL spectra acceptable quality of theoretical model cannot be achieved. This is illustrated in Fig. 3, where the PL model is 
a)

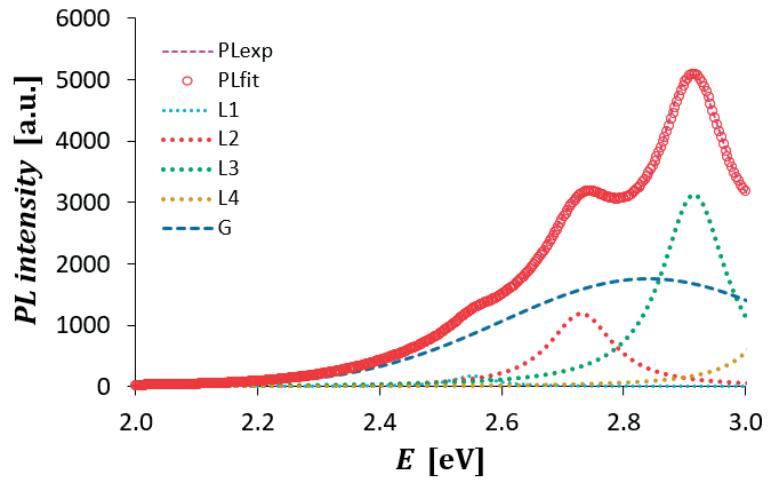

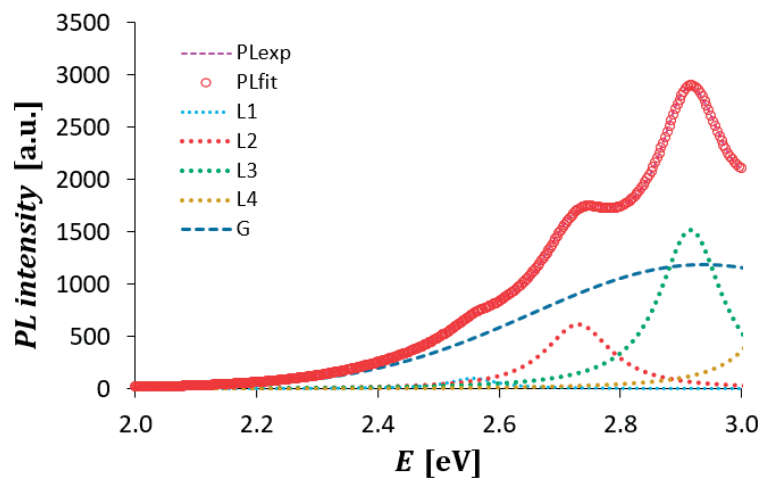

Fig. 2 Theoretical models of the PL spectra: a) $3.54 \mathrm{eV}$, b) $3.76 \mathrm{eV}$

constructed without the contribution of excited ncSi distribution. Disagreement between experimental spectrum (dashed line) and theoretical model (circles) can be clearly observed.

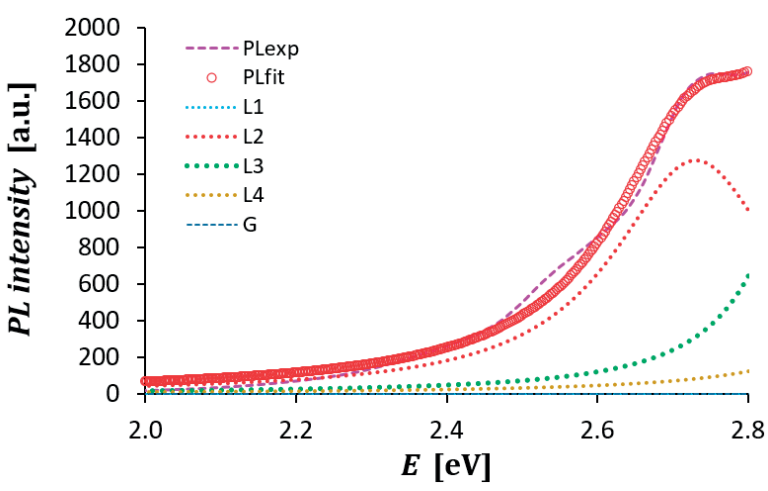

Fig. 3 Theoretical model of the ncSi-DMA PL experiment without contribution of the optical excitation of the $n c$ Si subsystem

In Fig. 4 two optimized theoretical models based on one Gaussian function and eight Lorentz functions for excitation energies a) $E_{e x}=4.00 \mathrm{eV}$ and b) $E_{e x}=4.28 \mathrm{eV}$ are shown.

a)

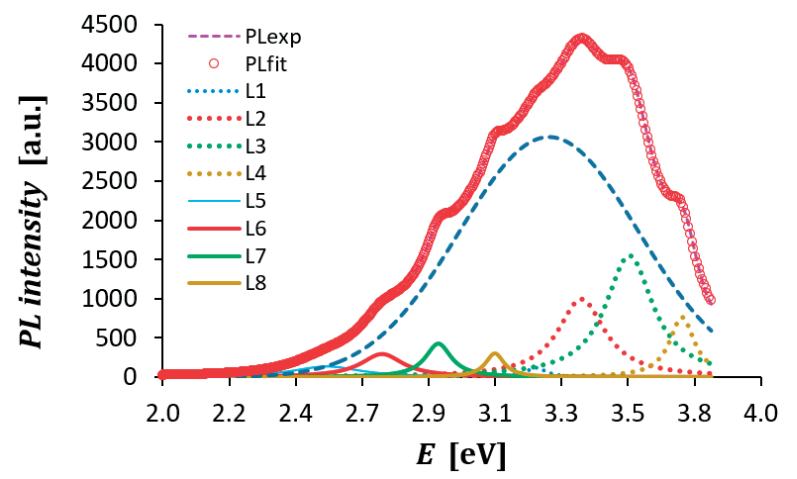

For these excitation energies the dominant contribution process to the PL spectrum is connected with the optical excitation of the ncSi distribution. The selected system of discrete energy levels contributing to the PL active transitions (8 Lorentz functions) and modelled contribution of the size distribution of ncSi nanocrystals (Gauss function) suitable describes observed PL spectrum.

Possible sizes of ncSi particles in nanocrystal subsystem are connected with the distribution of energy gaps due to the quantum shape effects. We estimated energy gap $E_{g}$ dependence of Si nanocrystal on its radius $R$ by using the equation [18]

$E_{g}=\frac{h^{2} \pi^{2}}{2 R^{2}}\left(\frac{1}{m_{e}^{*}}+\frac{1}{m_{h}^{*}}\right)-\frac{1.8 e^{2}}{\varepsilon R}$,

where $R$ is the radius of Si nanocrystal, $m_{e}^{*}$ and $m_{h}^{*}$ are effective masses of electron and holes, $e$ is electron charge, $\varepsilon$ is vacuum permittivity. Values of excitation energies used in PL experiments influence the fraction of ncSi size distribution potentially excited by these photons. This fraction is connected with the distribution of ncSi energy gaps $E_{g}$ determined by the quantum size effect according Equation 4. In Fig. 5 the energy gap $E_{g}$ in energy area covering excitation energies in our PL experiments is shown. From this estimation possible ncSi size distribution observed

Fig. 4 Theoretical models of the PL spectra: a) $4.00 \mathrm{eV}$, b) $4.28 \mathrm{eV}$ 
in the ncSi-DMA system can be determined. Dominant fraction of the ncSi subsystem formed by the beads milling method has radius in interval 5-6 $\mathrm{nm}$ in agreement with the $\mathrm{x}$-ray and TEM methods.

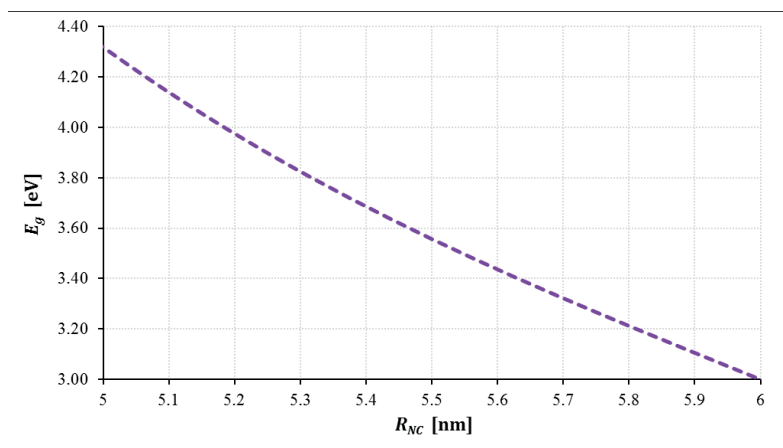

Fig. 5 Energy gap of $n c S i$

Optimized parameters of Gaussian component of the ncSiDMA PL theoretical model are shown in Table 1 and development of selected parameters is shown graphically in Fig. 6 .

With increasing excitation energy the position of Gaussian distribution centre shifts towards higher energies. It is connected with fraction of $\mathrm{Si}$ nanocrystals with suitable sizes in a given distribution, which can be excited by used photon energy in the PL experiment. The width of this component in Fig. 6b increases systematically with significant changes observed in the experiment with excitation energies above $4 \mathrm{eV}$. Larger amount of ncSi sizes in given distribution can be excited in agreement to available excitation photon energy.
Lorentzian components in the theoretical model of PL experiment demonstrated in Fig. 2 and Fig. 4 show stable values of theoretical peak positions. This is connected with the structure of energy levels of adsorbed DMA molecules and can be used for the study of possible excitation and transition processes from higher energy states as well as for study of DMA excitation caused by the charge transfer processes in the ncSi-DMA system.

\section{Conclusions}

Silicon nanocrystals were fabricated by the beads milling method from the Si swarf. Experimental PL spectra of the ncSiDMA system were analysed by modelling of possible excitation and charge transport processes. Properties of observed PL transitions were determined by using theoretical models optimized with the experimental PL spectra. From resulting theoretical model characteristics of PL transitions were determined: a) energy $(\mathrm{eV})$, intensity, and width of transitions between energy states in the ncSi-DMA donor-acceptor system based on Lorentz functions for modelling DMA photoluminiscence, and b) energy $(\mathrm{eV})$, intensity, and width of the Gaussian component modelling the contribution of the excitons confined in a size distribution of ncSi. Experimental PL spectra of the ncSi-DMA system cannot be explained by using only PL transitions in a DMA molecule. Energy and charge transport processes in the ncSi-DMA system substantially influence the shape of the observed PL spectra. PL contribution of the ncSi subsystem strongly depends on particle size distribution (due to presence of the $E_{g}$ distribution) and

Parameters of Gaussian function in optimized theoretical PL model

\begin{tabular}{ccccccc}
\hline$E_{\text {exc }}[\mathrm{eV}]$ & $\mathbf{3 . 0 2}$ & $\mathbf{3 . 1 8}$ & $\mathbf{3 . 5 4}$ & $\mathbf{3 . 7 6}$ & $\mathbf{4}$ & $\mathbf{4 . 2 8}$ \\
\hline $\boldsymbol{x}_{0}$ & 2.77103 & 2.87553 & 2.79019 & 2.95512 & 3.11901 & 3.62252 \\
$\sigma$ & 0.3642 & 0.25653 & 0.21812 & 0.28925 & 0.27125 & 0.47642 \\
$\boldsymbol{I}_{0 G}$ & 56.3661 & 1390.76 & 1547.41 & 1231.27 & 1670.84 & 2164.67 \\
\hline
\end{tabular}

a)

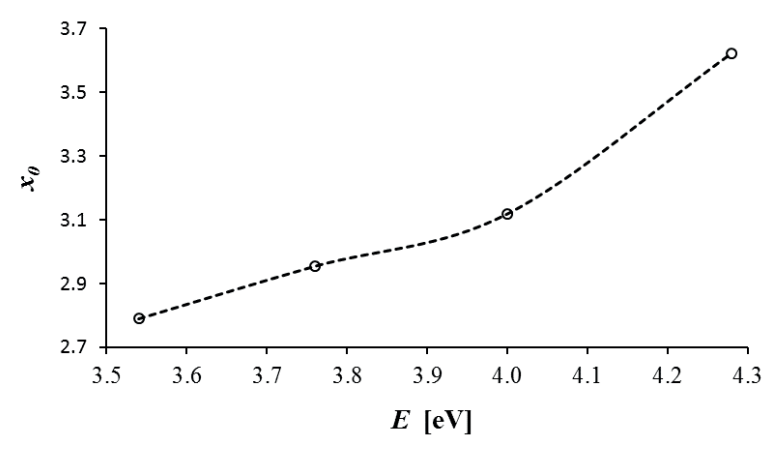

b)

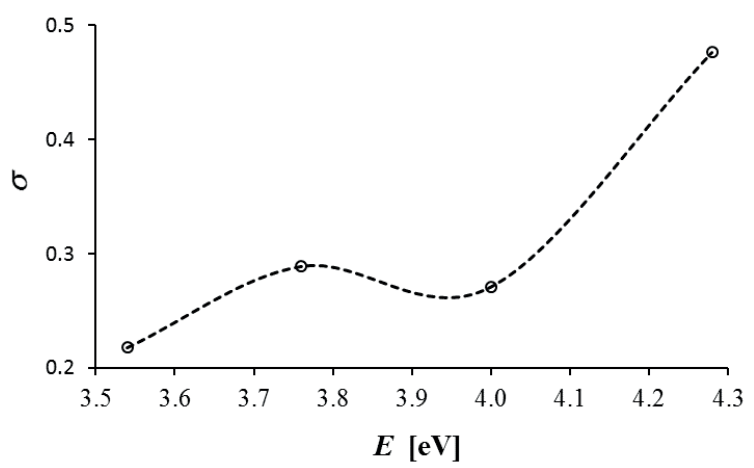

Fig. 6 Development of a) central position $x_{0}$ and $b$ ) width parameter $\sigma$ of Gaussian component of the PL theoretical model 
is shifted with increasing PL excitation energy. This shift also influences the PL transitions in the structure of energy levels of DMA molecule due to energy and charge transport mechanism in the ncSi-DMA system. The charge transfer process is based on substantial overlay of wavefunctions of two systems - DMA molecule and ncSi particle. It exponentially depends on the distance between these subsystems. This distance is modified during oxidation of ncSi particles and etching processes. This causes drastic quenching of PL transitions caused by the charge transfer mechanism.

\section{Acknowledgements}

This work was supported by grant of Science and Technology Assistance Agency APVV-15-0152, Scientific Grant Agency VEGA 1/0676/17 and 1/0076/15, Centre of Excellence of Power Electronics Systems and Materials ITMS 26220120003 and ITMS 26220120046, project ITMS 26210120021 and Japan Society for the Promotion of Science.

\section{References}

[1] EPUR, R., MINARDI, L. K., DATTA, M., CHUNG, S. J., KUMTA, P. N.: A Simple Facile Approach to Large Scale Synthesis of High Specific Surface Area Silicon Nanoparticles. Journal of Solid State Chemistry, 208, 93-98, 2013.

[2] BULUTAY, C., OSSICINI, S.: Electronic and Optical Properties of Silicon Nanocrystals. Pavesi, L., Turan, R. (Eds), Silicon Nanocrytals, Wiley-VCH Verlag GmbH \& Co, KGaA, Weinheim, 2010.

[3] EROgbogbo, F., LiN, T., TUCCIARONE, P. M., LAJOIE, K. M., LAI , L., LATKI, G. D., PRASAD, P. N., SWIHART, M. T.: On-Demand Hydrogen Generation Using Nanosilicon: Splitting Water without Light, Heat, or Electricity. Nano Letters, 13, 451456, 2013.

[4] O'FARREll, N., HOUlTON, A., HORROCKS, B. R.: Silicon Nanoparticles: Applications in Cell Biology and Medicine. International Journal of Nanomedicine, 1, 451-472, 2006.

[5] MATSUMOTO, T., MAEDA, M., FURUKAWA, J., KIM, W-B., KOBAYASHI, H.: Si Nanoparticles Fabricated from Si Swarf by Photochemical Method. Journal of Nanoparticle Research, 16, 2240-1-7, 2014.

[6] MAEDA, M., IMAMURA, K., MATSUMOTO, T., KOBAYASHI, H.: Fabrication of Si Nanoparticles from Si Swarf and Application to Solar Cells. Applied Surface Science, 312, 39-42, 2014.

[7] MAKIMURA, T., KUNII, Y., ONO, N., MURAKAMI, K.: Silicon Nanoparticles Embedded in $\mathrm{SiO}_{2}$ Films with Visible Photoluminescence. Applied Surface Science, 127-129, 388-92, 1988.

[8] Watanabe, K., SAWADA, K., KOSHIBA, M., FUJII, M., HAYASHI, S.: Photo-luminescence Decay Dynamics of Si Nanoparticles Prepared by Pulsed Laser Ablation. Applied Surface Science, 197-198, 635-638, 2002.

[9] LACONA, F., BONGIORNO, C., SPINELlA, C., BONINELlI, S., PRIOLO, F.: Formation and Evolution of Luminescent Si Nanoclusters Produced by Thermal Annealing of $\mathrm{SiO}_{\mathrm{x}}$ Films. Journal of Applied Physics, 95, 3723-3732, 2004.

[10] SALIVATI, N., AN, Y. Q., DOWNER, M. C., EKERDT, J. G.: Hot-Wire Chemical Vapor Deposition of Silicon Nanoparticles on Fused Silica. Thin Solid Films, 517, 3481-3483, 2009.

[11] FAUChet, P. M., RUAN, J., CHEN, H., PAVESI, L., NEGRO, L. D., CAZZANELI, M., EllimaN, R. G., SMITH, N., SAMOC, M., LUTHER-DAVIES, B.: Optical Gain in Different Silicon Nanocrystal Systems. Optical Materials, 27, 745-749, 2005.

[12] MORALES-SANCHEZ, A., LEYVA, K. M., ACEVES, M., BARRETO, J., DOMINGUEZ, C., LUNA-LOPEZ, J. A., CARRILO, J., PEDRAZA, J.: Photoluminescence Enhancement through Silicon Implantation on SRO-LPCVD Films. Materials Science and Engineering: B, 174, 119-122, 2010.

[13] CHOI, B. J., LEE, J. H., YATSUI, K., YANG, S. C.: Preparation of Silicon Nanoparticles for Device of Photoluminescence. Surface and Coatings Technology, 201, 50036, 2007.

[14] OHNO, K.: Simple Calculations of Franck-Condon Factors for Electronic Transition Bands of Polyacenes. Chemical Physics Letters, 53, 571-577, 1978.

[15] JURECKA, S.: Theoretical Model of the Physical System: Optimization by the Genetic Algorithm. Dritsas, I. (Ed.), Stochastic Optimization - Seeing the Optimal for the Uncertain, InTech, Vienna, 2011.

[16] MUllerova, J., SUTTA, P., JURECKA, S.: Thin Film Silicon in Photovoltaics: The Role of Structure and Microstructure. Communications - Scientific Letters of the University of Zilina, 8(1), 5-9, 2006.

[17] DEVORE, J. L.: Probability and Statistics for Engineering and the Sciences, $8^{\text {th }}$ ed. Cengage Learning, Boston, 2011.

[18] HUMMEL, R. E., WISSMANN, P.: Handbook of Optical Properties: Optics of Small Particles, Interfaces and Surfaces. CRC Press LLC, Boca Raton, 1997. 